\title{
Negotiating The Learning Curve In Endoscopic Skull Base Surgery: A Local ENT Perspective
}

\author{
Ranjan Raychowdhury, ${ }^{1}$ Sandip Chatterjee ${ }^{1}$
}

\section{Introduction}

$\underline{\text { ABSTRACT }}$

Skull base surgery, although recognised as an interdisciplinary specialty involving both Neurosurgery and Otolaryngology in the West, has been the domain of the Neurosurgeon in West Bengal. Several superspecialty healthcare facilities in the state are now actively promoting endoscopic skull base surgery.

Structured training

The Pittsburgh group has published a systematic training programme for skull base surgery. Paradigm shift

Following the work of several groups, most notably the Pittsburgh group in the USA, a paradigm shift from open to endoscopic anterior skull base surgery has occurred.

Learning curve

This paper discusses how the ENT surgeon may negotiate the learning curve in endoscopic skull base surgery in the context of the local resources available.

$\underline{\text { Kevwords }}$

Skull Base; Endoscopy; Learning Curve; Otolaryngology; Neurosurgery

$\mathrm{A}$ natomically and pathologically the base of the skull separates the specialties of Neurosurgery and Ear, Nose, Throat- Head \& Neck Surgery (ENT-HNS). Pathology in this region would fall to the neurosurgeon if primarily intracranial (pituitary adenoma, craniopharyngioma, aesthesioneuroblastoma); to the ENT surgeon if primarily extracranial (e.g. inverted papilloma, juvenile angiofibroma, squamous carcinoma) and to either specialty if primarily basicranial (fibrous dysplasia, osteoma, chordoma, chondrosarcoma) depending upon the presentation. Combined open surgical approaches were reserved for lesions which transgressed the skull base: the anterior cranio-facial resection for invasive cancers and aesthesioneuroblastoma ${ }^{1,2}$ and the various lateral approaches popularised by $\mathrm{Fisch}^{3}$ for large glomus tumours.

The French neurosurgeon, Guiot, ${ }^{4}$ in 1963 first suggested that the standard microsurgical transrhinoseptal approach to the pituitary might be usefully supplemented by endoscopy. More than 30 years later, following the invention of the Hopkins rod lens, Jho and Carrau in Pittsburgh described a purely endoscopic transsphenoidal approach to the pituitary. ${ }^{5,6}$ The ability of the rigid endoscope to access the anterior base of the skull trans-nasally, avoiding any external incision, craniotomy and brain retraction, with consequent reduction in post-operative morbidity, has revolutionised skull base surgery.

Skull base surgery, although recognised as an interdisciplinary specialty involving both Neurosurgery and Otolaryngology in the West, ${ }^{7}$ has been the domain of the Neurosurgeon in West Bengal. With increasing public awareness of endoscopic skull base surgery several superspecialty healthcare facilities in West Bengal are now actively promoting this technique, and ENT participation in recent national skull base meetings ${ }^{8}$

1 - The Park Clinic, Kolkata

Corresponding author:

Dr Ranjan Raychowdhury

email: kolkata_ent@hotmail.com 
testifies to the growing interest in this field amongst ENT specialists. Although the endoscopic approach has many advantages it comes with its own risks: peroperative neural and/or vascular damage may have devastating consequences for the patient. Safe surgery with excellent outcomes requires teamwork between the ENT specialist and his Neurosurgical colleagues, and there is a fairly steep learning curve to be negotiated before full competence is achieved.

\section{Structured Training in Endoscopic Skull Base Surgery}

The Pittsburgh group, who have been in the forefront of promoting this technique, have developed a modular scheme of training by which the budding endoscopic skull base surgeon can progress in a structured fashion along the learning curve. ${ }^{9}$ The schema is applicable irrespective of whether or not the trainee is from an otolaryngological or a neurosurgical background (Table I).

Successful negotiation of this training scheme presupposes an extended period of training (the North American ENT Residency lasts for 6 years with a further two years Fellowship period) in a Department which has a high throughput of skull base cases and expert faculty to supervise training. Given the much shorter duration of MS/DNB training here, the most one can hope to achieve within three years is Level II training,

Table I: Modular scheme of training developed by the Pittsburgh Group

\begin{tabular}{|c|c|c|}
\hline Level I & Sinus surgery & \\
\hline \multirow[t]{3}{*}{ Level II } & Advanced sinus surgery & \\
\hline & & Cerebrospinal fluid leak \\
\hline & & Intrasellar - sella, pituitary \\
\hline \multirow[t]{4}{*}{ Level III } & Extrasellar - sella, pituitary & \\
\hline & & Optic nerve decompression \\
\hline & & Intraorbital surgery \\
\hline & & Extradural skull base surgery \\
\hline \multirow[t]{9}{*}{ Level IV } & Intradural skull base surgery & \\
\hline & A. With cortical cuff & \\
\hline & & Transplanum \\
\hline & & Transcribriform \\
\hline & & Type I craniopharyngioma \\
\hline & & Intradural skull base surgery \\
\hline & B. Without cortical cuff & \\
\hline & & Type II/III craniopharyngioma \\
\hline & & Transclival, intradural \\
\hline \multirow[t]{2}{*}{ Level V } & Coronal plane, carotid dissection & \\
\hline & & Vascular surgery \\
\hline
\end{tabular}


that too in only a handful of well-equipped centres. Further expertise will have to be obtained during Senior Residency and initial Consultant period, by attending hands-on workshops and by Fellowships at Centres of Excellence, both within and outside the country.

\section{The Paradigm Shift}

From the neurosurgical point of view endoscopic skull base surgery demands an entirely new skill set - working off a two dimensional monitor image rather than traditional three dimensional microscopic surgery. The otolaryngologist may be forgiven for thinking that, with a background in Functional Endoscopic Sinus Surgery (FESS) and training in Head \& Neck oncosurgery, the learning curve will be easier, but in reality taking the endoscope to the skull base requires a complete paradigm shift:

1. FESS, by definition, is a minimally invasive procedure, aiming to preserve as much normal anatomy and mucosa as possible, whereas endoscopic skull base surgery requires wide access to the lesion, for which sinonasal mucosa and structures like the middle turbinate are sacrificed on a regular basis

2. Skull base neoplasms are usually dealt with by intra-capsular debulking followed, if possible, by extra-capsular excision with minimal margins in order to preserve cerebral and/or cranial nerve function, rather than by en bloc excision with a 1 $\mathrm{cm}$ margin.

3. In FESS the surgeon is used to holding the endoscope in one hand and an instrument in the other, using one nostril, and changing instruments as required. In endoscopic skull base surgery the surgeon may hold either an endoscope and a suction cannula, or two instruments, and work via one nostril whilst the other surgeon uses the other nostril, and both surgeons work together in harmony.

4. For FESS, the otolaryngologist requires and uses a CT scan of the nose and para-nasal sinuses as a road map for the procedure. In endoscopic skull base surgery, however, the patients' imaging usually consists of MRI scans.

5. Much pre-operative discussion is required with the neurosurgical colleagues - both surgeons must have a clear idea of the aim of the procedure and the endpoint of surgery. Reconstruction of the dural barrier must always be kept in mind; e.g., arterial supply of naso-septal flaps should be preserved even if the flaps are not used, as they may be needed for revision surgery.

6. No amount of technology can replace a sound concept of the three dimensional anatomy of the skull base. Image-guidance/Neuro-navigation will not make your surgery safer if you are venturing into the para-sellar or clival areas without a clear understanding of the anatomical landmarks and neuro-vascular structures present.

7. Intra-operative haemorrhage can be extremely difficult to manage endoscopically, especially if the Internal carotid artery (ICA) is damaged. When imaging suggests proximity of the lesion to the ICA all necessary equipment and material for haemostasis must be kept readily available.

\section{Finding the Way}

Given that endoscopic skull base surgery requires close co-operation between the otolaryngologist and the neurosurgeon the interested ENT surgeon must find a neurosurgeon, preferably of the same generation, who is equally enthusiastic. Attendance at cadaveric workshops as a team is an excellent way of building operative coordination. Membership of the Skull Base Surgery Society of India apart from providing a valuable network of similar minded colleagues, also carries access to short fellowships at centres of excellence within the country.

\section{Conclusion}

Conventional skull base surgery, although recognised as an interdisciplinary specialty involving both Neurosurgery and Otolaryngology in the West, has been the domain of the Neurosurgeon in West Bengal. Endoscopic skull base surgery is a rapidly expanding, multi-disciplinary specialty which is gaining popularity amongst both neurosurgeons and otolaryngologists. 
After a thorough grounding in endoscopic sinus surgery interested ENT surgeons must build a good working relationship with a neurosurgeon, and obtain further training by means of workshops and fellowships, to establish themselves in this field.

\section{References}

1. Smith RR, Kloop CT, Williams JM. Surgical treatment of cancer of the frontal sinus and adjacent areas. Cancer 1954;7: 991-4.

2. Cheesman AD, Lund VJ, Howard DJ. Craniofacial resection for tumours of the nose and paranasal sinuses. Head Neck Surg 1986; 8: 429-35.

3. Fisch U. Infratemporal fossa approach to tumours of the temporal bone and base of the skull. J Laryngol Otol. 1978;

\section{2:949-67}

4. Guiot G, Comoy C. Ventriculoscopy. Rev Prat. 1963;13:3655-6

5. Jho HD, Carrau RL, Ko Y, Daly MA. Endoscopic pituitary surgery: an early experience. Surg Neurol. 1997; 47: 213-22

6. Jho HD, Alfieri A. Endoscopic endonasal pituitary surgery: evolution of surgical technique and equipment in 150 operations. Minim Invasive Neurosurg 2001; 44:1-12

7. National Institute for Health and Clinical Excellence. Improving Outcomes for people with brain and other CNS tumours. Section 6. June 2006

8. Available at: http://www.sbssi.org/directory.htm

9. Snyderman CH, Pant H, Kassam AB, Carrau RL, Prevedello DM Gardner PA, The Learning Curve for Endonasal Surgery of the Cranial Base: A Systematic Approach to Training. In: Endoscopic Approaches to the Skull Base. Prog Neurol Surg. Basel, Karger, 2012, vol 26, pp 222-231 